\title{
Pill Counting in the Determination of Factors Affecting Medication Adherence in Patients with Type 2 Diabetes: A Retrospective Observational Study
}

\author{
Megumi Shiomi · Momoka Kurobuchi · Yoichi Tanaka • \\ Tesshu Takada $\cdot$ Katsuya Otori
}

Received: March 22, 2021 / Accepted: May 27, 2021 / Published online: June 13, 2021

(C) The Author(s) 2021

\begin{abstract}
Introduction: For medication adherence, pill counting has higher accuracy in objective assessment. However, previous reports have shown that factors such as psychological bias and other people's involvement in managing and helping patients take their medications may influence the outcomes. In Japan, all prescription medicines of patients are checked by medical reconciliation, and a pill count is performed during hospitalization. This study investigated factors affecting the medication adherence of patients with type 2 diabetes mellitus (T2DM), including patient- and medication-related factors, by pill counting using medical reconciliation in a situation where the patient's psychological bias is low.
\end{abstract}

M. Shiomi ( $₫) \cdot$ M. Kurobuchi · Y. Tanaka ·

K. Otori

Department of Clinical Pharmacy, School of Pharmacy, Kitasato University, 5-9-1 Shirokane, Minato-ku, Tokyo 108-8641, Japan

e-mail: shiomim@pharm.kitasato-u.ac.jp

M. Shiomi · K. Otori

Department of Pharmacy, Kitasato University

Medical Center, 6-100 Arai, Kitamoto, Saitama 364-

8501, Japan

T. Takada

Department of Endocrinology, Diabetes, and

Metabolism, School of Medicine, Kitasato

University, 1-15-1 Kitasato, Minami-ku, Sagamihara,

Kanagawa 252-0374, Japan
Methods: This study included 103 patients with T2DM who had been treated with oral hypoglycemic agents (OHAs) for at least 24 weeks. Patients whose OHAs were managed by another person were excluded. We calculated medication adherence values (MAVs) according to the following formula: MAV = (total prescription days - prescription days of OHAs brought when admitted)/(days from the start of OHAs to hospitalization). The relationship between MAVs and patient- and medication-related factors was analyzed.

Results: On multiple linear regression analysis of patient-related factors with $P<0.10$ in the univariate analysis as explanatory variables, a lower number of chronic diseases $(\beta=0.017$; $P<0.001)$ and higher number of OHAs ( $\beta=-0.021 ; P=0.04)$ were independent factors for lower MAV. Medication-related factors were not found to be independent factors.

Conclusions: Our findings suggest that poor adherence was independently associated with lower number of chronic diseases and higher number of OHAs in patients with T2DM.

Keywords: Low physical bias; Medical reconciliation; Medication adherence value; Medication adherence; Pill count; Selfadherence of patients; Type 2 diabetes mellitus 


\section{Key Summary Points}

\section{Why carry out this study?}

As type 2 diabetes mellitus (T2DM) medication adherence has been reported to be lower than that to other diseases, elucidating factors that influence adherence is important.

Previous reports might have been influenced by patients' psychological biases and may not have taken into account who is managing and helping patients take their medication. Therefore, the exact factors need to be clarified.

We investigated the factors affecting medication adherence in patients with T2DM using pill counting in a situation where the patient's psychological bias is low.

\section{What was learned from this study?}

Poor adherence was associated with lower number of chronic diseases and higher number of oral hypoglycemic agents.

Medication-related factors were nonindependent factors of adherence.

In addition, increasing the number of doses per day, taking medications at lunch, and $\alpha$-glucosidase inhibitors may be factors associated with poor adherence.

\section{DIGITAL FEATURES}

This article is published with digital features, including a summary slide, to facilitate understanding of the article. To view digital features for this article go to https://doi.org/10.6084/ m9.figshare.14681406.

\section{INTRODUCTION}

Diabetes is a chronic metabolic disease that increases the risk of micro- and macrovascular complications, such as retinopathy, nephropathy, neuropathy, stroke, and cardiovascular disease $[1,2]$. Long-term self-care is important to prevent the onset and progression of disabling complications of diabetes and to ensure a life expectancy that is similar to that of healthy people. Pharmacologic therapy is a key component in the treatment of diabetes, and oral hypoglycemic agents (OHAs) are very effective in managing type 2 diabetes mellitus (T2DM). However, adherence to diabetes medications has been reported to be lower than that to other disease medications [3]. A systematic review has shown that adherence to OHAs ranged from $36 \%$ to $93 \%$ in patients with diabetes [4]. Nonadherence to OHAs has been associated with higher glycated hemoglobin (HbA1C), increased risk of hospitalization, and high mortality [5, 6]. Therefore, medication adherence should be improved in patients with diabetes.

Several medication adherence measures can be potentially used in patients with chronic disease, such as subjective and objective measurements. Objective measurements are mainly used to assess OHAs. Several studies used the secondary database analysis, mainly proportion of days covered (PDC), medication possession ratio (MPR), and pill count as objective measurements [7]. OHA adherence studies using these measurements suggest that poor adherence-related factors vary, including patient characteristics, treatment regimens, patient's out-of-pocket costs, socioeconomic factors, and concomitant medications [8-13]. MPR and PDC are based on pharmacy claims data that accumulates medication usage data billed by pharmacies to insurance companies. Therefore, they only measure refilling behaviors and could not confirm whether the patient actually took the medication. Previous adherence studies of patients with T2DM have described this indirect measurement as a study limitation [8-11, 14]. Pill counting or medication event monitoring system (MEMS) has higher accuracy as 
compared to other methods [15]. However, pill count or MEMS performed to measure adherence is likely to result in psychological bias among patients. Patients who participated in pill counting or MEMS were aware that their medication intake was being monitored, which may have resulted in psychological bias for patients. Alternatively, patients may not return all the medicines to hide their misbehavior. MEMS also has the potential to activate the container without taking medication as it measures the patient's behavior in opening the package. Previous adherence studies, including pill counting and MEMS, have described these factors as limitations [16-18]. In addition, these objective studies do not investigate in detail who manages and helps patients in taking medications from home, i.e., the patients themselves, parents, spouse, children, home care worker, or others. Adherence rates and factors may vary depending on the person who manages the patient's medications. These factors, including not ascertaining whether patients actually take the medication, patients' psychological biases, and identifying who manages and helps the patient's medication, could be reasons for variations in the conclusion of adherence studies. A systematic review shows that associations between adherence and other factors were inconsistent among the reviewed studies [14]. However, the factors affecting medication adherence under more accurate conditions that account for this bias, i.e., the patient's self-adherence by pill counting in a situation where the patient's psychological bias is low, have not been investigated.

In Japan, at the time of hospitalization, all prescription medicines are checked by medical reconciliation and a pill count is performed. In addition, patients are also interviewed on who manages and helps them with the medication at home along with patient's information such as history of allergy and events effects at the time of medical reconciliation. The purpose of performing pill counting is to ensure that patients take the medications they bring with them to avoid prescribing new medications during hospitalization, and not to measure adherence. This system can be used to examine factors influencing patients' adherence to medication with less psychological bias than in a typical pill counting. This study investigated factors affecting patient's own medication adherence by pill counting using medical reconciliation, in a situation where the patient's psychological bias is low, using an assessment method that we defined as medication adherence values (MAVs).

\section{METHODS}

\section{Study Design and Participants}

This retrospective study included Japanese patients with T2DM who had been treated with OHAs and were subsequently hospitalized for any treatment at Kitasato University Medical Center between April 2015 and December 2019. Patients who could be followed up continuously from the start of OHA treatment to hospitalization in Kitasato University Medical Center were included. The reason for this is to clarify the detailed withdrawal process or temporary suspension of medication due to treatment or examination during the hospital visit. In our study, adherence was assessed by pill counting of the medications that patients brought with them at admission. Therefore, if their prescription was changed shortly before admission, adherence could be assessed over a short period of time; it is not possible to accurately assess patient's adherence over a short period of time. In addition, the type and frequency of medication may affect adherence. Hence, we included patients who had been taking the same OHAs for at least 24 weeks at the time of hospitalization. During patient interviews on admission, patients who declared that they manage the medication themselves at home, not by others such as family members and home care workers, were included. Patients aged less than 20 years, with visual or other physical impairment, diagnosed with cognitive impairments, and diagnosed with mental health diseases or received psychotropic agents as exclusion criteria were excluded. This study was approved by the ethical committee of Kitasato University Medical Center (registration number H30-009) and was conducted following 
the principles of the Declaration of Helsinki. Informed consent was obtained from all patients included in this study.

\section{Assessments}

We obtained the following patient characteristics from medical records: gender, age, duration of diabetes, body mass index (BMI), HbA1C, diabetes complications (nephropathy, retinopathy, and macrovascular complications), number of chronic diseases, number of OHAs, use of insulin, number of non-hypoglycemic agents, history of adverse events, family history of diabetes, taking dietary supplement, alcohol consumption, employment history, living condition, and marital status as patient-related factors. We defined nephropathy as a urine albumin-to-creatinine ratio of at least $30 \mathrm{mg} / \mathrm{g}$ creatinine and/or estimated glomerular filtration rate (eGFR) of less than $30 \mathrm{~mL} / \mathrm{min} / 1.73 \mathrm{~m}^{2}$ [19]. Retinopathy was determined according to the International Clinical Diabetic Retinopathy Severity Scale by a qualified ophthalmologist [20]. We defined retinopathy as mild nonproliferative diabetic retinopathy or more. Macrovascular complications include ischemic heart, cerebrovascular, and peripheral arterial diseases. Chronic disease was a co-existing medical condition in which the patient was diagnosed by a physician and regularly visited the physician to control the symptoms and signs, with or without taking medications. We defined non-hypoglycemic agents as regularly prescribed medications taken for at least 24 weeks. Alcohol consumption was defined as anyone who reported consuming alcoholic beverages at least 4 days a week. With regard to the marital status, only married and no single, separated, divorced, or widow status was considered. With regard to medication-related factors, prescription information of OHAs (dosing period, dosing time, number of doses, and types of OHAs) was obtained from medical history.

\section{Medication Adherence}

Kitasato University Medical Center asks all patients to bring all of their current prescription medications, including dosages, with them at the time of hospitalization because of two reasons. First, the list of medications should be checked by medical reconciliation to ensure that all the medications taken by the patient are as accurate as possible. Second, if patients need to continue taking the same type of medications during hospitalization, they can use them without a new prescription. For a newly hospitalized patient in Kitasato University Medical Center, the pharmacist usually interviews patients about their information such as history of allergy and adverse events, taking dietary supplements, and who manages and helps them in taking medications at home. The next steps include obtaining and verifying the patient's medication history, documenting the patient's medication history, and writing orders for the hospital medication regimen. During these steps, the pharmacist receives the patient's medications and verifies their prescription and pill count. Before the hospital admission, patients are told by the physician, nurse, and clerks to bring all their medications from home. After hospitalization, the pharmacist checks again to confirm if there are any medications left at home and asks them to bring the residual medications. Using this system, medication adherence was assessed using the total number of prescription days obtained from prescription history and the number of OHA prescription days brought in at the hospital, calculated by pill counting to assess medication adherence. To assess the medication adherence, the following formula, defined as MAVs, has been used: (total prescription days - prescription days of OHAs brought when admitted)/(days from the start of OHAs to hospitalization). We considered the date of the first filled OHA prescription as the index date. Moreover, the total number of prescription days was the sum of the number of OHA prescription days from the first to the last prescription (latest prescription before admission). Patients with MAVs of 1 are those who never missed taking OHAs from the start of prescription to the hospitalization. As the number of days of missed doses increases, i.e., as adherence decreases, MAVs decrease. Patients with MAV $>1$ were excluded from our study, as there was a possibility that they may 
have overdosed medicines or not brought all medications to the hospital. Patients who reported that they had lost medications or did not bring all medications were also excluded.

\section{Statistical Analysis}

Data were expressed as mean and standard deviation or numbers and percentages. To examine factors related to medication adherence to OHAs therapy, factors were examined by dividing them into patient- and medicationrelated, since some patients had been taking more than one type of OHA. Univariate analysis was performed to assess the association between MAVs and patient-related or MAVs and medication-related factors as explanatory variables. Stepwise multiple linear regression analysis was performed to examine factors affecting medication adherence. MAVs were also included as dependent variables. The values with $P<0.10$ on univariate analysis were included as explanatory variables. With the analysis of patient background factors, if a patient was taking more than one type of OHA, the lower adherence value was used as the dependent variable. Multicollinearity of variables was assessed using the variance inflation factor (VIF), with serious collinearity defined as VIF $\geq 10$. All statistical analyses were performed using Easy R (Saitama Medical Center, Jichi Medical University, Japan), a modified version of $\mathrm{R}$ commander designed to add statistical functions frequently used in biostatistics [21], and $P$ values of less than 0.05 were considered significant.

\section{RESULTS}

\section{Patient Characteristics}

In total, 4317 patients were eligible to participate in this study. However, 4205 were excluded because of no continuous follow-up in Kitasato University Medical Center, not taking the same OHAs for at least 24 weeks, or someone other than the patient managing and helping the medication. Nine patients who met our exclusion criteria were also excluded. Finally, 103 patients were included in this study (Fig. 1). None of the 103 patients reported that they did not bring all their medications or had lost them. Table 1 presents patient characteristics and clinical information. Of them, 56.3\% were men, with a mean age of $64.1 \pm 13.0$ years: almost half of the patients (48.5\%) were aged less than 65 years; and the mean $\mathrm{HbA} 1 \mathrm{C}$ was $9.1 \pm 1.9 \%$. With regard to complications, $59.2 \%$ had nephropathy and $11.7 \%$ had kidney failure with eGFR $<30 \mathrm{~mL} / \mathrm{min} / 1.73 \mathrm{~m}^{2}$ [19]; $40.8 \%$ and $7.8 \%$ of patients had retinopathy and proliferative retinopathy, respectively [20]. No patient had macular edema [20]; $24.3 \%$ of patients had macrovascular complications. The mean number of chronic diseases, OHAs, and non-hypoglycemic agents were $3.3 \pm 2.1,2.2 \pm 1.0$, and $5.4 \pm 4.0$, respectively. In addition, $55.3 \%$ had used insulin as concomitant medication. With regard to adverse events, $21.4 \%$ had hypoglycemia from the start of administration of hypoglycemic agents to hospitalization.

\section{Prescription Information of OHAs and MAVs}

The total number of OHAs taken by the patients was 230. Table 1 shows the prescription information of all OHAs. The mean dosing period was $108.4 \pm 76.1$ weeks and $24.9 \%$ were less than 48 weeks, $33.5 \%$ were between 48 and 96 weeks, and $41.6 \%$ were more than 96 weeks. The highest number of doses per day was once a day at $62.4 \%$. OHAs were most frequently taken at breakfast $(94.2 \%)$. The most frequently prescribed type of OHAs was dipeptidyl peptidase 4 inhibitors (DPP4) (38.7\%), followed by metformin (25.4\%) and sulfonylureas (13.3\%). The mean MAV was $0.94 \pm 0.10$. About $76.3 \%$ of all OHAs had a MAV of at least 0.9. MAV of at least 0.8 but less than 0.9 was observed in $11.0 \%$ of all OHAs. The MAV was less than 0.8 in $12.7 \%$ of all OHAs.

\section{Patient-Related Factors Affecting MAVs}

Table 2 shows patient characteristics and clinical information that were associated with MAVs 


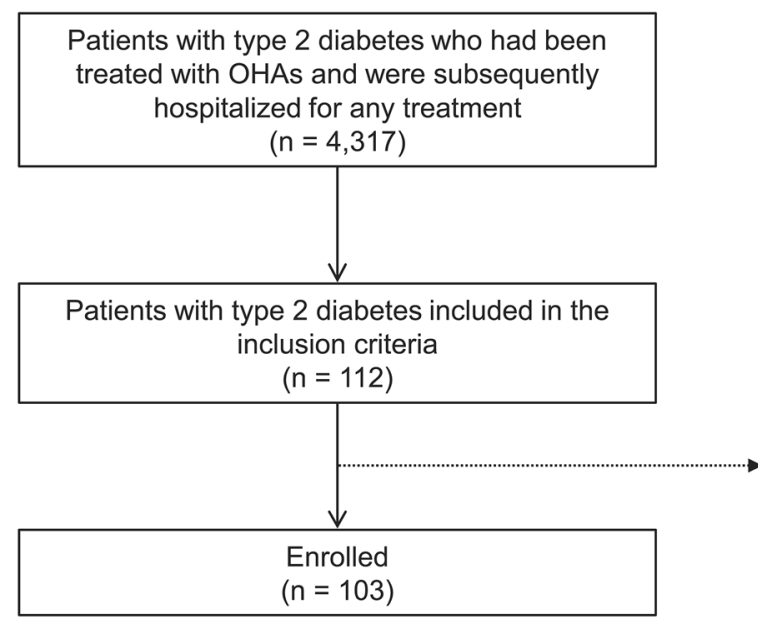

Fig. 1 Flow diagram of patients involved in this study. Inclusion criteria are as follows: continuous follow-up in Kitasato University Medical Center, taking the same

in the univariate linear regression analysis. Patients with lower MAV had significantly lower numbers of chronic diseases $(\beta=0.017$; $P<0.001)$, fewer non-hypoglycemic agents ( $\beta=0.009 ; P<0.001)$, and were more likely to be employed $(\beta=-0.049 ; P=0.02)$. Patients with lower MAV also tended to have higher HbA1C $\quad(\beta=-0.010 ; \quad P=0.07), \quad$ lesser macrovascular disease $(\beta=0.044 ; P=0.07)$, and higher number of OHAs $(\beta=-0.021 ; P=0.05)$. Age, history of adverse events, and living conditions were not associated with MAVs. A stepwise multiple linear regression analysis was performed using factors with $P<0.10$ in univariate linear regression analysis as explanatory variables. All models had VIF values of less than 10. In the stepwise multiple linear regression analyses, a lower number of chronic diseases $(\beta=0.017 ; P<0.001)$ and higher number of OHAs $(\beta=-0.021 ; P=0.04)$ were significantly associated with poor medication adherence in patients with T2DM treated with OHAs (Table 2).

\section{Medication-Related Factors Affecting MAVs}

Table 3 shows the patients' prescription information associated with MAVs in the univariate linear regression analysis. Patients with lower
Excluded $(n=9)$

Under the age of $20(n=1)$

Visual or other physical impairment $(n=1)$

Cognitive impairments $(n=3)$

Mental health diseases or received psychotropic agent $(n=2)$

MAVs of $>1(n=2)$

OHAs for at least 24 weeks, or patients themselves manage their medication. MAVs medication adherence values, OHAs oral hypoglycemic agents

MAVs tended to take higher number of doses per day $(\beta=-0.018 ; P=0.05)$, more lunchtime doses $(\beta=-0.030 ; P=0.08)$, and more patients taking $\alpha$-glycosidase inhibitors $(\beta=-0.050$; $P=0.06)$. Dosing period and OHAs administered before meals were not associated with MAVs. A stepwise multiple regression analysis was performed using factors with $P<0.10$ in univariate linear regression analysis as explanatory variables. All models had VIF values of less than 10. The stepwise multiple regression analysis did not identify any significant factors affecting medication adherence.

\section{DISCUSSION}

This study showed that the number of chronic diseases and OHAs are factors associated with medication adherence among patients with T2DM. This finding suggests that medication adherence becomes better as the number of chronic diseases increases. In addition, this finding also suggests that medication adherence becomes worse as the number of OHAs increases. The patients' lifestyle did not affect their medication adherence. We accurately assessed medication adherence by pill counting in addition to prescription history. We took into account periods of withdrawal due to surgery or 
Table 1 Patient characteristics, clinical, and prescription information $(n=103)$

Patient characteristics and clinical information

$$
\begin{aligned}
& \text { Male (\%) } \\
& \text { Age (\%) } \\
& 20-64 \text { years } \\
& 65-74 \text { years } \\
& \geq 75 \text { years }
\end{aligned}
$$

Duration of diabetes (\%)

$$
\begin{aligned}
& <10 \text { years } \\
& 10-19 \text { years } \\
& \geq 20 \text { years } \\
& \text { BMI }(\%) \\
& <25 \mathrm{~kg} / \mathrm{m}^{2} \\
& \geq 25 \mathrm{~kg} / \mathrm{m}^{2} \\
& \text { HbA1C }(\%)
\end{aligned}
$$

$9.1 \pm 1.9$

Complications of diabetes (\%)

$$
\text { Nephropathy }
$$

Retinopathy

Macrovascular disease

Number of chronic disease

Number of OHAs

Insulin therapy (\%)

$3.3 \pm 2.1$

$2.2 \pm 1.0$

55.3

Number of non-hypoglycemic agents

$5.4 \pm 4.0$

Adverse events (\%)

Adverse events related to hypoglycemic agent (\%)

Hypoglycemia

Others

Family history of diabetes (\%)

Current drinking (\%)

Dietary supplement (\%)

Employed (\%)

Living alone (\%)

Marital status (\%)

In-hospital prescription (\%)
Table 1 continued

Prescription information of OHAs

Total number of OHAs 230

Dosing period of the same OHAs (\%)

$<48$ weeks

48-96 weeks

$>96$ weeks

Number of doses/day (\%)

Once

Twice

Three times

OHAs administered before meals (\%)

Administration of OHAs (\%)

At breakfast

At lunch

At dinner

Types of OHAs (\%)

DPP4 inhibitors

Metformin

Sulfonylureas

$\alpha$-Glycosidase inhibitors

SGLT2 inhibitors

5.8

Glinides

3.5

Data are expressed as the mean \pm standard deviation, numbers, or percentages

$B M I$ body mass index, DPP4 dipeptidyl peptidase 4, OHAs oral hypoglycemic agents, SGLT2 sodium glucose cotransporter 2

tests to assess adherence more accurately. In order to assess adherence in more detail, only patients who self-administered their medication were included. Factors related to their living environment that might affect adherence were also taken into account. To the best of our knowledge, this is the first study to accurately 
Table 2 Patient-related factors of MAVs as identified via univariate and multiple linear regression analyses

\begin{tabular}{|c|c|c|c|c|c|c|}
\hline \multirow[t]{2}{*}{ Variable } & \multicolumn{3}{|c|}{ Univariate } & \multicolumn{3}{|l|}{ Multiple } \\
\hline & $\bar{\beta}$ & $95 \% \mathrm{CI}$ & $P$ value & $\bar{\beta}$ & $t$ value & $P$ value \\
\hline Age $(20-64,65-74, \geq 75$ years $)$ & 0.020 & $-0.004,0.044$ & 0.10 & & & \\
\hline Duration of diabetes $(<10,10-19, \geq 20$ years $)$ & 0.007 & $-0.019,0.032$ & 0.59 & & & \\
\hline $\mathrm{BMI}\left(<25, \geq 25 \mathrm{~kg} / \mathrm{m}^{2}\right)$ & -0.030 & $-0.072,0.011$ & 0.15 & & & \\
\hline HbA1C (\%) & -0.010 & $-0.021,0.001$ & 0.07 & & & \\
\hline \multicolumn{7}{|l|}{ Complications of diabetes } \\
\hline Nephropathy & 0.019 & $-0.024,0.061$ & 0.38 & & & \\
\hline Retinopathy & 0.002 & $-0.040,0.044$ & 0.92 & & & \\
\hline Macrovascular disease & 0.044 & $-0.003,0.092$ & 0.07 & & & \\
\hline Number of chronic diseases & 0.017 & $0.007,0.026$ & $<0.001$ & 0.017 & 3.585 & $<0.001$ \\
\hline Number of OHAs & -0.021 & $-0.041,0.0001$ & 0.05 & -0.021 & -2.129 & 0.04 \\
\hline Insulin therapy $(\%)$ & 0.029 & $-0.012,0.070$ & 0.17 & & & \\
\hline Number of non-hypoglycemic agents & 0.009 & $0.004,0.014$ & $<0.001$ & & & \\
\hline Adverse events & -0.006 & $-0.048,0.037$ & 0.80 & & & \\
\hline \multicolumn{7}{|l|}{ Adverse events related hypoglycemic agents } \\
\hline Hypoglycemia & -0.006 & $-0.057,0.045$ & 0.81 & & & \\
\hline Others & -0.024 & $-0.069,0.021$ & 0.29 & & & \\
\hline Family history of diabetes & -0.005 & $-0.048,0.038$ & 0.82 & & & \\
\hline Current drinking & -0.043 & $-0.100,0.013$ & 0.13 & & & \\
\hline Dietary supplement & -0.033 & $-0.089,0.023$ & 0.24 & & & \\
\hline Employed & -0.049 & $-0.091,-0.007$ & 0.02 & & & \\
\hline Living alone & 0.035 & $-0.027,0.098$ & 0.26 & & & \\
\hline Marital status & -0.019 & $-0.063,0.026$ & 0.40 & & & \\
\hline
\end{tabular}

Measurements were carried out using univariate and multiple liner regression analyses Adjusted $R^{2}=0.13, P<0.001$

$\beta$ standard regression coefficient, $B M I$ body mass index, $C I$ confidence interval, $M A V s$ medication adherence values, $O H A s$ oral hypoglycemic agents

investigate factors affecting the medication adherence of patients with T2DM in a situation where the patient's psychological bias is low.

A previous study reported that medication adherence was significantly higher in patients with co-existing illness than in those without in patients with hypertension, a chronic disease similar to T2DM [22]. DiMatteo et al. showed that nonadherence is more than 1.5 times higher in patients who do not perceive a disease severity threat, and the odds of adherence were almost 2.5 times higher if patients believe that the disease to be prevented or treated is a severe and life-threatening [23]. Furthermore, patients who were rated poorer in health by their physicians are more adherent to treatment [23]. 
Table 3 Medication-related factors of MAVs as identified via univariate linear regression analyses

\begin{tabular}{lccc}
\hline Variable & \multicolumn{2}{l}{ Univariate } & \\
\cline { 2 - 4 } & $\boldsymbol{\beta}$ & $\mathbf{9 5 \%} \mathbf{C I}$ & $\boldsymbol{P}$ value \\
\hline Dosing period (in 24-week increments) & 0.015 & $-0.004,0.033$ & 0.13 \\
Number of doses/day & -0.018 & $-0.036,0.0002$ & 0.05 \\
OHAs administered before meals & -0.012 & $-0.050,0.026$ & 0.54 \\
Administration of OHAs & & & \\
At breakfast & -0.010 & $-0.075,0.054$ & 0.75 \\
At lunch & -0.030 & $-0.065,0.004$ & 0.08 \\
At dinner & -0.024 & $-0.055,0.007$ & 0.12 \\
Types of OHAs & & & \\
DPP4 inhibitors & 0.021 & $-0.009,0.052$ & 0.17 \\
Metformin & -0.013 & $-0.048,0.021$ & 0.44 \\
Sulfonylureas & -0.015 & $-0.059,0.029$ & 0.51 \\
$\alpha$-Glycosidase inhibitors & -0.050 & $-0.103,0.003$ & 0.06 \\
SGLT2 inhibitors & 0.020 & $-0.045,0.084$ & 0.55 \\
Glinides & 0.033 & $-0.049,0.115$ & 0.42 \\
\hline
\end{tabular}

Measurements were carried out using univariate liner regression analysis

$\beta$ standard regression coefficient, $C I$ confidence interval, DPP4 dipeptidyl peptidase 4, $M A V s$ medication adherence values, $O H A s$ oral hypoglycemic agents, $S G L T 2$ sodium glucose cotransporter 2

In our study, patients with other chronic diseases in addition to T2DM were more aware of their illnesses and had higher medication adherence than patients with T2DM. The increase in illnesses may also increase the opportunity to interact with physicians, one of the factors that lead to an increased awareness of the disease severity and improve adherence. It may be important for health care providers to educate patients with only T2DM (or few other comorbidities) about the disease and to convey a strong message that good medication adherence affects the maintenance of glycemic control, prevention of complications, and reduction of mortality. Low health literacy has been shown to be associated with poorer healthrelated knowledge and comprehension and is a factor in health outcomes [24]. In patients with T2DM, higher health literacy has been shown to be associated with greater diabetes-specific knowledge [25]. It may also be necessary to improve health literacy to increase awareness of the disease and effective patient self-management. Conversely, adherence has been reportedly increased with lower comorbidity depending on the type of comorbidity [26]. The type of comorbidity has also been reported to affect medication adherence [14, 27, 28]. Depression is an important risk factor for poor patient adherence [14, 28]; however, mental health diagnoses or those receiving psychotropic agents were excluded in this study. In addition, other comorbidities were not evaluated in detail because of varied comorbidities. To further clarify the results, we may provide more specific insight into factors that affect adherence in patients with T2DM by categorizing them according to comorbidity.

It has been reported that the higher the number of OHAs, the lower the adherence 
[29, 30]. Al-Qazaz et al. [29] showed that monotherapy was associated with higher diabetes knowledge and higher adherence compared to combination therapy. Melikian et al. [30] reported that patients receiving monotherapy with an OHA exhibited significantly greater adherence compared with patients on combination therapy. We consider two possible reasons why medication adherence may decrease as the number of OHAs increases. Melikian et al. also reported that adding a second OHA to patients treated with OHA monotherapy resulted in a significant decrease in adherence [30]. In addition, they reported a significant improvement in adherence rates in patients who had been receiving combination therapy and were switched to fixed-dose combination therapy [30]. Therefore, these considerations suggest that increased OHA burden may have led to decreased medication adherence. Another reason could be a decline in disease awareness. Patients with T2DM have been reported to have difficulties in recognizing their symptoms, and a clear lack of awareness on the risk of diabetes-related complications was observed among patients with T2DM [31, 32]. Since risk perception plays an important role in behavioral change, if risk perception is lacking, the possibility of performing the expected behavior may be low. Although patients included in our study had diabetes complications, the number of patients with proliferative retinopathy and renal failure was small. Multiple OHAs may have been prescribed to manage poor glycemic control caused by poor adherence due to the patient's lack of awareness of the presence of diabetes. Since these discussions are influenced by the psychological background of the patient, a combination of subjective and objective assessments is needed to examine the factors that influence medication adherence in patients with T2DM in further detail.

This study showed that medication-related factors were not associated with medication adherence. However, a lower number of medication doses per day was associated with higher adherence, whereas lunchtime medication and $\alpha$-glycosidase inhibitors were associated with lower adherence. A previous study reported that once-daily dosing had higher adherence than twice-daily dosing [33]. In addition, other studies reported that once-daily dosing intervals were associated with higher adherence compared to twice-daily and three times per day intervals $[34,35]$. Therefore, medication adherence may be improved by reducing the number of doses. Work and going out may have been associated with poor medication intake at lunch. Medication adherence may decrease as a result of the time constraint during lunchtime or because of not carrying the medication with them when they go out. Several studies have investigated the relationship between $\alpha$-glycosidase inhibitors and adherence [36, 37]. Hertz et al. [36] reported that initial treatment using an $\alpha$-glucosidase inhibitor was a risk factor for early non-persistence and discontinuation over time. Aoki et al. [37] reported that $48 \%$ of patients with T2DM reported forgetting to take it at least once a week in an interview of patients prescribed with $\alpha$-glycosidase inhibitors. In addition, as $\alpha$-glycosidase inhibitors should be taken three times per day and at lunch, they may be associated with poor adherence. Since majority of these previous studies have been followed for at least 8-12 months, long-term evaluation is needed.

This study has limitations. First, although physicians, pharmacists, nurses, and clerks explain to patients that they should bring all of their medications from home before and during admission, we can only trust the patient as to whether or not they brought all their medicines. Therefore, medication adherence is overestimated if the patient does not bring all remaining medications on admission. In addition, if a patient does not declare lost medication and overdose, estimates of medication adherence may be inaccurate. For this reason, we excluded patients with MAV $>1$ because it is highly possible that they did not bring all their medications to the hospital or overdosed. For the other patients, we could only trust their selfreporting. Second, we included patients with T2DM who had been hospitalized for any treatment and who took the same OHAs for at least 24 weeks at the time of hospitalization. Thus, hospitalization itself or exclusion of patients who had not been taking the same OHA for 24 weeks could be a bias. Finally, the 
medical insurance system in Japan is a universal health insurance system in which all Japanese are enrolled in some form of public medical insurance. Although there is little bias in the study patient characteristics, this difference in the insurance system may have influenced the differences in factors compared to previous reports.

\section{CONCLUSIONS}

This study highlighted that poor adherence was independently associated with a smaller number of chronic diseases and increasing number of OHAs in patients with T2DM. In addition, increasing the number of doses per day, taking at lunch, and $\alpha$-glucosidase inhibitors may be factors of poor adherence. Our pill counting was more accurate because data were based on the patient's self-management of medication with less psychological bias, excluding withdrawal periods due to surgery or testing and including the patient's environment. Our findings help better understand the factors of poor adherence affecting patients with T2DM in actual clinical practice: providing an environment and support that allows patients with T2DM to continue treatment even if they are unaware of their disease can contribute to achieving a good HbA1C and reducing the occurrence of complications.

\section{ACKNOWLEDGEMENTS}

We thank the participants of the study.

Funding. No funding or sponsorship was received for this study or publication of this article. The Rapid Service Fee was funded by the authors.

Authorship. All named authors meet the International Committee of Medical Journal Editors criteria for authorship for this article, take responsibility for the integrity of the work as a whole, and have given their approval for this version to be published.
Author Contributions. MS initiated and designed the study, interpreted findings, and drafted the manuscript. KM interpreted findings and conducted the statistical analysis. YT designed the study and reviewed the manuscript. TT designed the study and reviewed the manuscript. KO designed the study and reviewed the manuscript. All authors participated in the writing of the manuscript.

Disclosure. Megumi Shiomi, Momoka Kurobuchi, Yoichi Tanaka, Tesshu Takada, and Katsuya Otori declare that they have nothing to disclose.

Compliance with Ethics Guidelines. This study was approved by the ethical committee of Kitasato University Medical Center (registration number H30-009) and was conducted following the principles of the Declaration of Helsinki. Informed consent was obtained from all patients included in this study.

Data Availability. The datasets generated during and/or analyzed during the current study are available from the corresponding author on reasonable request.

Prior Presentation. Some portion of this work was presented at the 80th American Diabetes Association Scientific Sessions in June 2020 in Chicago. However, the target patients, evaluation, and statistical analysis methods were different.

Open Access. This article is licensed under a Creative Commons Attribution-NonCommercial 4.0 International License, which permits any non-commercial use, sharing, adaptation, distribution and reproduction in any medium or format, as long as you give appropriate credit to the original author(s) and the source, provide a link to the Creative Commons licence, and indicate if changes were made. The images or other third party material in this article are included in the article's Creative Commons licence, unless indicated otherwise in a credit line to the material. If material is not included in the article's Creative Commons licence and your intended use is not permitted by statutory 
regulation or exceeds the permitted use, you will need to obtain permission directly from the copyright holder. To view a copy of this licence, visit http://creativecommons.org/licenses/by$\mathrm{nc} / 4.0 /$.

\section{REFERENCES}

1. Roussel R, Steg PG, Mohammedi K, Marre M, Potier L. Prevention of cardiovascular disease through reduction of glycaemic exposure in type 2 diabetes: a perspective on glucose-lowering interventions. Diabetes Obes Metab. 2018;20:238-44.

2. Stratton IM, Adler AI, Neil HA, et al. Association of glycaemia with macrovascular and microvascular complications of type 2 diabetes (UKPDS 35): prospective observational study. BMJ. 2000;321: 405-12.

3. DiMatteo MR. Variations in patients' adherence to medical recommendations: a quantitative review of 50 years of research. Med Care. 2004;42:200-9.

4. Cramer JA. A systematic review of adherence with medications for diabetes. Diabetes Care. 2004;27: 1218-24.

5. Egede LE, Gebregziabher M, Echols C, Lynch CP. Longitudinal effects of medication nonadherence on glycemic control. Ann Pharmacother. 2014;48: 562-70.

6. Ho PM, Rumsfeld JS, Masoudi FA, et al. Effect of medication nonadherence on hospitalization and mortality among patients with diabetes mellitus. Arch Intern Med. 2006;166:1836-41.

7. Lam WY, Fresco P. Medication adherence measures: an overview. Biomed Res Int. 2015;2015:217047.

8. Tunceli K, Zhao C, Davies MJ, et al. Factors associated with adherence to oral antihyperglycemic monotherapy in patients with type 2 diabetes. Patient Prefer Adherence. 2015;9:191-7.

9. Kirkman MS, Rowan-Martin MT, Levin R, et al. Determinants of adherence to diabetes medications: findings from a large pharmacy claims database. Diabetes Care. 2015;38:604-9.

10. Curkendall SM, Thomas N, Bell KF, Juneau PL, Weiss AJ. Predictors of medication adherence in patients with type 2 diabetes mellitus. Curr Med Res Opin. 2013;29:1275-86.
11. Garcia ML, Castañeda SF, Allison MA, Elder JP, Talavera GA. Correlates of low-adherence to oral hypoglycemic medications among Hispanic/Latinos of Mexican heritage with type 2 diabetes in the United States. Diabetes Res Clin Pract. 2019;155: 107692.

12. Katz LL, Anderson BJ, McKay SV, et al. Correlates of medication adherence in the TODAY cohort of youth with type 2 diabetes. Diabetes Care. 2016;39: 1956-62.

13. Asche C, LaFleur J, Conner C. A review of diabetes treatment adherence and the association with clinical and economic outcomes. Clin Ther. 2011;33:74-109.

14. Krass I, Schieback P, Dhippayom T. Adherence to diabetes medication: a systematic review. Diabet Med. 2015;32:725-37.

15. Farmer KC. Methods for measuring and monitoring medication regimen adherence in clinical trials and clinical practice. Clin Ther. 1999;21:1074-90.

16. van Onzenoort HA, Verberk WJ, Kessels AG, et al. Assessing medication adherence simultaneously by electronic monitoring and pill count in patients with mild-to-moderate hypertension. Am J Hypertens. 2010;23:149-54.

17. Takahara M, Shiraiwa T, Ogawa N, Katakami N, Matsuoka TA, Shimomura I. Clinical backgrounds associated with discrepancy between subjective and objective assessments of medication adherence in Japanese type 2 diabetic patients. Diabetol Int. 2016;7:398-403.

18. Niechciał E, Acerini CL, Chiesa ST, et al. Medication adherence during adjunct therapy with statins and ACE inhibitors in adolescents with type 1 diabetes. Diabetes Care. 2020;43:1070-6.

19. Haneda M, Utsunomiya K, Koya D, et al. A new classification of diabetic nephropathy 2014: a report from joint committee on diabetic nephropathy. Clin Exp Nephrol. 2015;19:1-5.

20. Wilkinson CP, Ferris FL 3rd, Klein RE, et al. Proposed international clinical diabetic retinopathy and diabetic macular edema disease severity scales. Ophthalmology. 2003;110:1677-82.

21. Kanda Y. Investigation of the freely available easyto-use software "EZR" for medical statistics. Bone Marrow Transplant. 2013;48:452-8.

22. Mizuno R, Fujimoto S, Uesugi A, et al. Influence of living style and situation on the compliance of taking antihypertensive agents in patients with essential hypertension. Intern Med. 2008;47: 1655-61. 
23. DiMatteo MR, Haskard KB, Williams SL. Health beliefs, disease severity, and patient adherence: a meta-analysis. Med Care. 2007;45:521-8.

24. Berkman ND, Sheridan SL, Donahue KE, Halpern DJ, Crotty K. Low health literacy and health outcomes: an updated systematic review. Ann Intern Med. 2011;155:97-107.

25. Caruso R, Magon A, Baroni I, et al. Health literacy in type 2 diabetes patients: a systematic review of systematic reviews. Acta Diabetol. 2018;55:1-12.

26. Rolnick SJ, Pawloski PA, Hedblom BD, Asche SE, Bruzek RJ. Patient characteristics associated with medication adherence. Clin Med Res. 2013;11: 54-65.

27. Zanders MM, Haak HR, van Herk-Sukel MP, van de Poll-Franse LV, Johnson JA. Impact of cancer on adherence to glucose-lowering drug treatment in individuals with diabetes. Diabetologia. 2015;58: 951-60.

28. Lin EH, Katon W, Von Korff M, et al. Relationship of depression and diabetes self-care, medication adherence, and preventive care. Diabetes Care. 2004;27:2154-60.

29. Al-Qazaz H, Sulaiman SA, Hassali MA, et al. Diabetes knowledge, medication adherence and glycemic control among patients with type 2 diabetes. Int J Clin Pharm. 2011;33:1028-35.

30. Melikian C, White TJ, Vanderplas A, Dezii CM, Chang E. Adherence to oral antidiabetic therapy in a managed care organization: a comparison of monotherapy, combination therapy, and fixed- dose combination therapy. Clin Ther. 2002;24: 460-7.

31. Rouyard T, Kent S, Baskerville R, Leal J, Gray A. Perceptions of risks for diabetes-related complications in type 2 diabetes populations: a systematic review. Diabet Med. 2017;34:467-77.

32. van Puffelen AL, Heijmans MJ, Rijken M, et al. Illness perceptions and self-care behaviours in the first years of living with type 2 diabetes; does the presence of complications matter? Psychol Health. 2015;30:1274-87.

33. Dezii CM, Kawabata H, Tran M. Effects of oncedaily and twice-daily dosing on adherence with prescribed glipizide oral therapy for type 2 diabetes. South Med J. 2002;95:68-71.

34. Paes AH, Bakker A, Soe-Agnie CJ. Impact of dosage frequency on patient compliance. Diabetes Care. 1997;20:1512-7.

35. Ayoub D, Mroueh L, El-Hajj M, et al. Evaluation of antidiabetic medication adherence in the lebanese population: development of the lebanese diabetes medication adherence scale. Int J Pharm Pract. 2019;27:468-76.

36. Hertz RP, Unger AN, Lustik MB. Adherence with pharmacotherapy for type 2 diabetes: a retrospective cohort study of adults with employer-sponsored health insurance. Clin Ther. 2005;27: 1064-73.

37. Aoki K, Nakamura A, Ito S, et al. Administration of miglitol until 30 min after the start of a meal is effective in type 2 diabetic patients. Diabetes Res Clin Pract. 2007;78:30-3. 